\section{ORIGINAL RESEARCH}

\author{
L. Pierot \\ X. Leclerc \\ A. Bonafé \\ S. Bracard, for the \\ French Matrix \\ Registry Investigators
}

\title{
Endovascular Treatment of Intracranial Aneurysms with Matrix Detachable Coils: Midterm Anatomic Follow-Up from a Prospective Multicenter Registry
}

\begin{abstract}
BACKGROUND AND PURPOSE: Polyglycolic/polylactic acid-covered platinum coils have been proposed to reduce the rate of aneurysm recanalization after endovascular treatment. A prospective and multicenter registry was conducted in France to evaluate the safety and short-term and midterm efficacy of Matrix coils. This analysis focused on anatomic midterm results.
\end{abstract}

\begin{abstract}
MATERIALS AND METHODS: Two hundred thirty-six patients harboring 244 ruptured or unruptured aneurysms treated via endovascular approach by using Matrix coils were included in this registry. Treatment was totally or partially performed by using Matrix coils. Anatomic results were evaluated on postoperative and last-follow-up digital subtraction angiography (DSA) by using the Raymond scale. "Recanalization" was defined as worsening, and "progressive thrombosis" was defined as improvement on the Raymond scale.
\end{abstract}

\begin{abstract}
RESULTS: Anatomic midterm follow-up was obtained in 165 of 236 patients (70\%) harboring 171 aneurysms (range, 6-27 months; mean, $14 \pm 4$ months). At midterm follow-up angiography, 79 aneurysms were completely occluded (46.2\%), 43 had a neck remnant (25.1\%), and 49 had an aneurysm remnant $(28.7 \%)$. Of 171 aneurysms, recanalization was observed in 44 patients $(25.7 \%)$, including major recanalization in 18 patients $(10.5 \%)$. Recanalization was more frequent if the embolized volume of aneurysm was $\leq 25 \%$. Progressive thrombosis was observed in 52 aneurysms (30\%). No bleeding or rebleeding was observed during the period of follow-up.
\end{abstract}

CONCLUSION: The efficacy of Matrix coils in preventing recanalization was not demonstrated in our series. In agreement with previous studies using bare platinum coils, volumic occlusion was an important feature for the prediction of aneurysm recanalization. A high percentage of progressive thrombosis in incompletely treated aneurysms was observed in our series, suggesting a biologic activity of Matrix coils.

E ndovascular treatment by using bare platinum coils is currently used in patients worldwide as an alternative to surgery to occlude ruptured and unruptured cerebral aneurysms. ${ }^{1}$ The most significant limitation of the technique is aneurysm recanalization that may occur in $15 \%-30 \%{ }^{2-5}$ and may potentially lead to aneurysm rebleeding. The modification of the surface of bare platinum coils was proposed in the late 1990s to accelerate the biologic response to coils and subsequently reduce the rate of recanalization. ${ }^{6-7}$

The first coated coil available for clinical use was covered with a bioactive copolymer consisting of polyglycolic/polylactic acid (PGLA). ${ }^{7}$ Several monocentric series have been recently published regarding the short- and midterm clinical and anatomic results of Matrix detachable coils (Boston Scientific, Natick, Mass) in the treatment of cerebral aneurysms. ${ }^{8-12} \mathrm{~A}$ small number of patients were included in most series (25-112 patients), and midterm clinical and anatomic results were not always available.

\section{Received February 5, 2007; accepted after revision May 31}

From the Department of Neuroradiology (L.P.), Reims University, Reims, France; the Department of Neuroradiology (X.L.), Lille University, Lille, France; the Department of Neuroradiology (A.B.), Montpellier University, Montpellier, France; and the Department of Neuroradiology (A.B.), Nancy University, Nancy, France.

This registry was financially supported by Boston Scientific France. L. Pierot is consultant to Boston Scientific France.

Please address correspondence to Laurent Pierot, MD, Service de Radiologie, Hôpital Maison-Blanche, 45, rue Cognacq-Jay, 51092 Reims cedex; e-mail: Ipierot@chu-reims.fr DOI 10.3174/ajnr.A0738
We recently published the immediate clinical and anatomic posttreatment results of a large prospective multicenter registry conducted in France in $2004 .^{13}$ In this series, the overall morbidity and mortality rates of patients with intracranial aneurysms treated with Matrix detachable coils were within the ranges of previously published series using bare platinum coils. Similar results were reported by other teams. ${ }^{8-12}$

The potential influence of PGLA platinum coils on anatomic results in the time course after endovascular treatment of intracranial aneurysms remains debated. In a preliminary study, Kang et $\mathrm{al}^{8}$ found that the recanalization rate by the use of PGLA-coated coils was similar to that previously reported with the use of bare platinum coils. More recently, Niimi et $\mathrm{al}^{12}$ found a higher recanalization rate with Matrix coils, whereas Murayama et $\mathrm{al},{ }^{11}$ in the largest series published, to our knowledge, showed that anatomic results at midterm follow-up were better with Matrix coils compared with the Guglielmi detachable coil (GDC, Boston Scientific) system.

The objective of the present study was to evaluate the midterm anatomic results in 165 patients harboring 171 intracranial aneurysms treated by Matrix coils and included in a prospective multicenter French registry.

\section{Methods}

Population, Protocol, and Immediate Results

A prospective and multicenter registry including 261 patients (163 women and 98 men; age range, $18-78$ years; mean, 48.5 years) with 
ruptured and unruptured intracranial aneurysms treated by Matrix coils was conducted in France between January and October 2004. Inclusion criteria were patients harboring ruptured or unruptured aneurysms $<25 \mathrm{~mm}$. Fusiform and dissecting aneurysms were excluded as well as aneurysms associated with brain arteriovenous malformations. Patients could be included as long as the first coil implanted for the treatment was a Matrix coil. Use of bare platinum coils was possible. Use of any other bioactive coil was considered a protocol violation. The goal of this registry was, first, to assess the safety and efficacy of PGLA-covered platinum coils and, second, to evaluate long-term anatomic results. Immediate posttreatment clinical and angiographic results in 236 patients (149 women and 87 men; age range, 21-78; mean age, 48.7 years) harboring 244 aneurysms have already been evaluated and published. ${ }^{13}$

\section{Anatomic Follow-Up}

Anatomic results were evaluated on digital subtraction angiography (DSA), including selective contrast injections and multiple projections. The degree of aneurysmal occlusion was defined by using the simplified 3-point classification scale: complete occlusion, neck remnant, and aneurysm remnant. ${ }^{5}$

For each patient, postembolization and follow-up angiograms were interpreted by 2 independent experienced neuroradiologists. In case of disagreement between the observers, a third neuroradiologist was involved. Postembolization and midterm follow-up angiograms were separately evaluated. No direct comparison of initial and midterm angiographic results was conducted.

"Recanalization" and "progressive occlusion" were defined as a change in the Raymond classification, comparing the rate of occlusion postembolization and on midterm follow-up. "Recanalization" was defined as a worsening of classification: minor recanalization, from complete occlusion to neck remnant or from neck remnant to aneurysm remnant; and major recanalization, from complete occlusion to aneurysm remnant. "Progressive occlusion" was defined as improvement of classification.

Recanalization and progressive occlusion were analyzed according to several anatomic and procedural features: size of the aneurysm, size of the neck, percentage of Matrix coils used (length of coils), and embolized volume of aneurysms.

According to Tamatani et al, ${ }^{14}$ embolized volume was calculated by using the following equation: volume of the embolized coil/volume of the aneurysm.

\section{Bleeding or Rebleeding/Retreatment}

Bleeding or rebleeding occurrence during the period of follow-up was evaluated. Retreatment of aneurysms during the period of follow-up was also recorded.

\section{Data Analysis}

Clinical and anatomic data were collected and entered via an electronic Website (www.kikamedical.com; Kika-Medical, Nancy, France).

Statistical analysis was independently conducted by Kika-Medical. According to the analysis regarding factors affecting anatomic results, the event was the recanalization, and the statistical unit was the aneurysm. The $\chi^{2}$ test was performed to evaluate the difference between the groups. Univariate and multivariate analysis was performed by using the logistic regression analysis to define the independent contribution of each prognostic factor with a $P$ value $<.20$ for the univariate analysis. Multivariate predictors were chosen by a step- wise procedure by using an entry criterion of 0.20 with a stay criterion of 0.10 . Statistical significance was declared if the 2 -sided $P$ value was $<.05$. All statistical analyses were performed by using the Statistical Package for the Social Sciences System for Windows (Version 11.5; SPSS, Chicago, Ill).

\section{Results}

\section{Anatomic Results}

Among the 236 patients (244 aneurysms) who were initially included in the registry, 14 were dead at 1 year, 14 were lost to follow-up, and 4 refused any follow-up. Finally, 204 patients had a clinical follow-up. In 6 patients, no angiographic follow-up was performed at 1 year due to patient's age in most cases. In 33 patients, angiograms were provided by the investigating center but were deemed insufficient by the reviewing neuroradiologists to evaluate precisely the rate of occlusion of aneurysms. These patients were, therefore, excluded from analysis.

Finally, among the 236 patients ( 244 aneurysms) who were initially included in the registry, 165 (171 aneurysms) had midterm anatomic follow-up by DSA 6-27 months after treatment (mean, $14 \pm 6$ months). In this group, clinical presentation was subarachnoid hemorrhage in 95 patients (57.6\%). In 70 patients (42.4\%), aneurysms were unruptured. Localizations were carotid siphon in 81 cases $(47.4 \%)$, anterior cerebral artery (ACA) and anterior communicating artery (AcomA) in 50 cases (29.2\%), middle cerebral artery (MCA) in 26 cases $(15.2 \%)$, and posterior circulation in 14 cases (8.2\%). Aneurysmal size was $\leq 10 \mathrm{~mm}$ in 143 cases $(83.6 \%)$ and $>10 \mathrm{~mm}$ in 28 cases $(16.4 \%)$. The size of the neck was $\leq 4$ $\mathrm{mm}$ in 141 cases $(82.5 \%)$ and $>4 \mathrm{~mm}$ in 30 cases $(17.5 \%)$. Dome-to-neck ratio was $\leq 1.5$ in 61 aneurysms $(35.7 \%)$ and $>1.5 \%$ in 110 aneurysms $(64.3 \%)$. An adjunctive technique was used in 50 cases (29.2\%); a remodelling technique, in 44 cases $(25.7 \%)$; and stent placement, in 6 cases $(3.5 \%)$. The percentage of Matrix coils used for the treatment (according to the length of coils) was $100 \%$ in 101 cases (59.1\%), 71\%-99\% in 19 cases $(11.1 \%)$, and $<70 \%$ in 51 cases $(29.8 \%)$. After treatment, embolized volume of aneurysm was $\leq 25 \%$ in 78 cases $(45.6 \%)$ and $>25 \%$ in 93 cases $(54.4 \%)$.

Anatomic evaluation at immediate postoperative phase and at midterm follow-up is reported in Table 1.

Recanalization and progressive occlusion are reported in Table 2. Aneurysm recanalization was observed in 44 aneurysms $(25.7 \%)$. Progressive occlusion was observed in 52 aneurysms $(30.4 \%)$.

The rate of recanalization and progressive occlusion was analyzed according to anatomic and procedural features. Recanalization rates were not significantly different according to aneurysm location: $23.4 \%$ for carotid siphon aneurysms, $30 \%$ for ACA and AcomA aneurysms, 23.1\% in the MCA group, and $28.6 \%$ in the posterior circulation group. Recanalization was significantly higher $(P=.001)$ in aneurysms $>10 \mathrm{~mm}(14$ cases, $50 \%$ ) than in aneurysms $\leq 10 \mathrm{~mm}$ (30 cases, $21 \%$ ). Recanalization was observed in 33 cases (24\%) in which the neck was $\leq 4 \mathrm{~mm}$ and in 11 cases (36\%) in which the neck was $4 \mathrm{~mm}$ (not significant). Recanalization was observed in 16 cases (26.2\%) in which the dome-to-neck ratio was $\leq 1.5$ and in 28 cases $(25.5 \%)$ in which the dome-to-neck ratio was $>1.5$ (not 


\begin{tabular}{|c|c|c|c|c|c|c|c|c|c|}
\hline & & \multicolumn{6}{|c|}{ Midterm Occlusion } & & \\
\hline & & \multicolumn{2}{|c|}{$\begin{array}{l}\text { Complete } \\
\text { Occlusion }\end{array}$} & \multicolumn{2}{|c|}{ Neck Remnant } & \multicolumn{2}{|c|}{$\begin{array}{c}\text { Aneurysm } \\
\text { Remnant }\end{array}$} & \multicolumn{2}{|c|}{ Total } \\
\hline & & No. & $\%$ & No. & $\%$ & No. & $\%$ & No. & $\%$ \\
\hline \multirow[t]{3}{*}{ Initial Occlusion } & Complete occlusion & 41 & 54.7 & 16 & 21.3 & 18 & 24.0 & 75 & 43.9 \\
\hline & Neck remnant & 22 & 48.9 & 13 & 28.9 & 10 & 22.2 & 45 & 26.3 \\
\hline & Aneurysm remnant & 16 & 31.4 & 14 & 27.5 & 21 & 41.2 & 51 & 28.9 \\
\hline Total & & 79 & 46.2 & 43 & 25.1 & 49 & 28.7 & 171 & 100 \\
\hline
\end{tabular}

Table 2: Recanalization and progressive occlusion in ruptured and unruptured groups

\begin{tabular}{|c|c|c|c|c|c|c|c|c|}
\hline & \multicolumn{4}{|c|}{ Recanalization } & \multicolumn{4}{|c|}{ Progressive Occlusion } \\
\hline & \multicolumn{2}{|c|}{ Minor } & \multicolumn{2}{|c|}{ Major } & \multicolumn{2}{|c|}{ Minor } & \multicolumn{2}{|c|}{ Major } \\
\hline & No. & $\%$ & No. & $\%$ & No. & $\%$ & No. & $\%$ \\
\hline Ruptured & 17 & 17.2 & 10 & 10.1 & 21 & 21.2 & 4 & 4.0 \\
\hline Unruptured & 9 & 12.5 & 8 & 11.1 & 15 & 20.8 & 12 & 16.7 \\
\hline Total & 26 & 15.2 & 18 & 10.5 & 36 & 21.1 & 16 & 9.4 \\
\hline
\end{tabular}

significant). Recanalization was observed in 17 cases (34\%) in which $>70 \%$ of Matrix coils (length of coils) were used and in 27 cases $(23 \%)$ when $<70 \%$ of Matrix coils were used (not significant). Recanalization was observed in 26 cases $(33 \%)$ if the embolized volume of aneurysm was $\leq 25 \%$ and in 18 cases $(19 \%)$ if the embolized volume was $>25 \%(P=.037)$.

No factor was significantly associated with progressive thrombosis.

\section{Bleeding/Rebleeding}

In the group of 204 patients having a 1-year clinical follow-up, no bleeding or rebleeding was observed.

\section{Retreatment}

In the period of follow-up, retreatment was performed in 13 of 171 aneurysms $(7.6 \%)$. Retreatment was performed $\leq 1$ year after the initial treatment in 8 patients and $>1$ year after treatment in 5 patients.

\section{Discussion}

Matrix detachable coils must be critically evaluated because they have been designed to improve the quality of long-term aneurysmal occlusion. Findings in previous series are contradictory. ${ }^{8-12}$ Two points are very important: Is the safety profile of Matrix coils similar to that if GDC coils? Are the midterm and long-term anatomic results improved by the use of Matrix detachable coils?

\section{Safety of the Matrix Detachable Coils}

According to the series dealing with the short-term results of the endovascular treatment (EVT) of intracranial aneurysms with Matrix coils, the safety profile of this device is satisfactory. ${ }^{8-13}$ According to our analysis of the largest cohort of patients treated with Matrix coils, the treatment-related mortality rates with Matrix coils were $0.8 \%$ and permanent morbidity, $2.5 \%$. Similar results were reported by Niimi et al, ${ }^{12}$ with procedural morbidity and mortality rates, respectively, $1.4 \%$ and $1.4 \%$. These results are not different from those reported in the largest series dealing with the EVT with bare platinum coils. In these series, the procedure-related morbidity and mortality rates were between $1.5 \%$ and $4 \% .{ }^{13}$

\section{Midterm Anatomic Results of EVT with Matrix Coils}

The definition of recanalization remains a controversial subject. In the first series dealing with midterm or long-term follow-up of intracranial aneurysms treated by EVT with GDC, percentages of recanalization were reported but the authors did not provide any definition of aneurysm recanalization. The progressive understanding that the stability of the occlusion of intracranial aneurysms after EVT is a key point has led to the development of new devices and to a more and more precise analysis of recanalization. In our series, we have defined a recanalization as a worsening of anatomic results according to the Raymond classification. This way of evaluating recanalization has some advantages and disadvantages. Postoperative and midterm angiographies can be separately and independently analyzed without comparison between angiograms. Moreover, in large series of patients, this method is relatively simple to use, especially if an independent core laboratory is dedicated for image interpretation However, this method includes limitations. As outlined by Niimi et al, ${ }^{12}$ aneurysm recanalization may occur without a change in the Raymond classification and especially for aneurysm remnants. For this reason, other definitions of recanalization have been used in the literature. Any increase in contrast filling on follow-up angiograms by using multiple views was considered by Byrne et $\mathrm{al}^{2}$ and Niimi et $\mathrm{al}^{12}$ as a recanalization. For the University of California, Los Angeles group, recanalization was defined as an increase in contrast filling of the aneurysm of $>10 \%,{ }^{4}$ whereas the anatomic result was judged as unchanged in case of contrast filling $<10 \%$. The main drawback of this method is performing an accurate evaluation of contrast filling.

Our midterm results are difficult to compare with those of previous series reporting midterm anatomic results after EVT with bare platinum coils ${ }^{2-5}$ due to population and methodology heterogeneity, but the overall recanalization rate reported in our series is in the same range.

Few results have been published regarding the midterm anatomic results after EVT with Matrix coils. In the series of Linfante et al, ${ }^{9}$ only 11 patients had 12-month follow-up, and no recanalization was observed. In the series of Niimi et al, ${ }^{12}$ follow-up angiographic results were obtained in 47 aneurysms 
treated with Matrix coils, and the overall recanalization rate was $57.4 \%$. For aneurysms treated with $>50 \%$ Matrix, the recanalization rate was $54.2 \%$. Recanalization of large aneurysms treated with $>50 \%$ Matrix was very high $(81.8 \%)$.

In the largest published series, ${ }^{11}$ follow-up angiograms after a mean of 8 months' treatment showed a stability of aneurysm occlusion in $51.7 \%$ of patients, a progressive thrombosis in $28.7 \%$, and a recanalization in $19.5 \%$. No recanalization was observed in cases of aneurysms completely occluded with Matrix coils at immediate postoperative control. A neck remnant was observed in 15\%, and a residual aneurysm, in 38\%.

Our series deals with the largest number of aneurysms with midterm follow-up angiograms obtained at a mean of 14 months after treatment. Overall recanalization was reported in $25.7 \%$ of patients. In $10.5 \%$ of patients, aneurysm recanalization was classified as major, and in $15.2 \%$, as minor. Our results are close to those reported by Niimi et al. ${ }^{12}$ In their series, according to our definition of recanalization, $27.7 \%$ of aneurysms treated with Matrix coils showed worsening of classification. Progressive occlusion was observed in a high percentage of cases $(30.5 \%)$ in agreement with the series of Murayama et al. ${ }^{11}$

\section{Factors Affecting Recanalization of Aneurysms Treated with Matrix Coils}

Anatomic and procedural features were statistically analyzed to depict the most important factors affecting recanalization. Recanalization rates were not statistically different in ruptured and unruptured groups (respectively, 27\% and 24\%). The recanalization rate was significantly higher in large aneurysms ( $50 \%$ versus $21 \%$ in the small aneurysm group). There was a trend toward a higher recanalization rate in case of an aneurysmal neck $4 \mathrm{~mm}$ in diameter (36\% versus $24 \%$ in aneurysms with a small neck), but this was not statistically significant. A similar nonsignificant result was obtained according to dometo-neck ratio: Aneurysm recanalization was observed in $26.2 \%$ in which the dome-to-neck ratio was $\leq 1.5$ and in $25.5 \%$ if it was $>1.5$.

Apart from the size of the aneurysm, the most significant factor affecting the rate of recanalization was the percentage of initial volumic occlusion of the aneurysm. Recanalization rates were respectively $33 \%$ and 19\% in case of initial volumic occlusion $\leq 25 \%$ and $>25 \%$, respectively. These findings are in agreement with previous studies showing a significant correlation between embolized volume and stability of aneurysm occlusion treated with bare platinum coils. ${ }^{14}$

As previously suggested by Niimi et $\mathrm{al}^{12}{ }^{12}$ this point is certainly important for the evaluation of midterm anatomic results with Matrix coils. Due to the friction of Matrix coils, the initial volumic occlusion of aneurysms treated with them is probably lower than that observed with bare platinum coils as demonstrated in a rabbit-elastase model ${ }^{15}$ dealing with angiographic and histologic analyses. In this study, the volumic occlusion was $31 \%$ with bare platinum coils and 23\% with Matrix coils, and despite the enhanced tissue response achieved with Matrix coils, the angiographic outcome was not improved by the use of the Matrix device compared with bare platinum coils. The absorbable nature of the bioactive coating of the Matrix coil (70\% by volume) may potentially contribute, in the time course after treatment, to decreasing the volu- mic occlusion. Thus, we have to keep in mind that packing attenuation remains the main factor for midterm and longterm anatomic results of aneurysms treated with coils and that the mechanical quality of Matrix coils remains the key point to decreasing the recanalization rate. The most recent version of the Matrix coil is theoretically associated with less friction and will probably improve mid- and long-term anatomic results.

\section{Bleeding/Rebleeding}

No bleeding or rebleeding was observed in our series during the period of follow-up, but some patients were lost for followup. Moreover, our group was heterogeneous, including ruptured and unruptured aneurysms. In previous series, rehemorrhage was observed in 1 of 62 patients $^{12}$ and in 1 of 103 patients. ${ }^{11}$ Overall the rate of rebleeding was between $0 \%$ and $1.6 \%$.

\section{Retreatment}

Thirteen aneurysms (7.6\%) were retreated during the period of follow-up. This is quite different from the retreatment rate $(21.3 \%)$ reported by Niimi et $\mathrm{al}^{12}$ but very close to the retreatment rate reported in previous reports using bare platinum coils. ${ }^{2,3,5,16}$ However, the retreatment rate is not a parameter that may be used to evaluate the midterm and long-term anatomic results because indications of retreatment are not clearly established and may differ according to the teams. Moreover, in our series, some patients were lost to follow-up, creating another bias.

\section{Conclusion}

The safety of endovascular treatment of intracranial aneurysms using Matrix coils was previously demonstrated in short-term results. ${ }^{13}$ The efficacy of Matrix coils to prevent aneurysm recanalization was not demonstrated in the present study because the rates of recanalization and retreatment were similar to those previously reported with bare platinum coils. However, our results demonstrated that volumic occlusion was the most important feature for the prediction of aneurysm recanalization, in agreement with previous studies using bare platinum coils. Recent improvement of the Matrix coil has lead to a decrease of the friction of the device, which will probably be associated with an increase of packing attenuation that may potentially improve long-term anatomic results. Most interesting, a high percentage of progressive thrombosis in incompletely treated aneurysms was observed in our series, suggesting a biologic activity of Matrix coils.

\section{Appendix}

\section{Participating Centers and Investigators:}

CHU Larrey, Angers, France; Anne Pasco

CHU Pellegrin, Bordeaux, France; Xavier Barreau, Jérôme Berge

CHU de la Côte de Nacre, Caen, France; Patrick Courthéoux CHU Gabriel Montpied, Clermont Ferrand, France; Emmanuel Chabert, Jean Gabrillargues

CHU Roger Salengro, Lille, France; Xavier Leclerc

CHU Neurologique, Lyon, France; Francis Turjman

CHU Gui de Chauliac, Montpellier, France; Alain Bonafé

CHU Hôpital Neurologique, Nancy, France; Serge Bracard 
CHU G et R. Laënnec, Nantes, France; Hubert Desal

CHU Saint-Roch, Nice, France; Mustapha Dib, Jacques Sedat

CHU La Pitié Salpêtrière, Paris, France; Alessandra Biondi,

Nader Sourour

CHU Lariboisière, Paris, France; Emmanuel Houdart

CHU de la Milétrie, Poitiers, France; Jacques Drouineau

CHU Maison Blanche, Reims, France; Laurent Pierot

CHU Purpan, Toulouse, France; Christophe Cognard

CHU Bretonneau, Tours, France; Denis Herbreteau

\section{References}

1. Molyneux A, Kerr R, Stratton I, et al, and the International Subarachnoid Aneurysm Trial (ISAT) Colaborative Group. International Subarachnoid Aneurysm Trial (ISAT) of neurosurgical clipping versus endovascular coiling in 2143 patients with ruptured intracranial aneurysms: a randomised trial. Lancet 2002;360:1262-63

2. Byrne JV, Sohn MJ, Molyneux A. Five-year experience in using coil embolization for ruptured intracranial aneurysm: outcomes and incidence of late rebleeding. J Neurosurg 1999;90:656-63

3. Cognard C, Weill A, Spelle L, et al. Long-term angiographic follow-up of 169 intracranial berry aneurysms occluded with detachable coils. Radiology 1999;212:348-56

4. Murayama Y, Nien LN, Duckwiler G, et al. Guglielmi detachable coil embolization of cerebral aneurysms: 11 years' experience. J Neurosurg 2003;98:959-66

5. Raymond J, Guilbert F, Weill A, et al. Long-term angiographic recurrences after selective endovascular treatment of aneurysms with detachable coils. Stroke 2003;34:1398-403

6. Murayama Y, Vinuela F, Tateshima S, et al. Bioabsorbable polymeric material coils for embolization of intracranial aneurysms: a preliminary experimental study. J Neurosurg 2001;94:454-63

7. Murayama Y, Tateshima S, Gonzalez NR, et al. Matrix and bioabsorbable polymeric coils accelerate healing of intracranial aneurysms; long-term experimental study. Stroke 2003;34:2031-37

8. Kang HS, Han MH, Kwon BJ, et al. Short-term outcome of intracranial aneurysms treated with polyglycolic acid/lactide copolymer-coated coils compared to historical controls treated with bare platinum coils: a single-center experience. AJNR Am J Neuroradiol 2005;26:1921-28

9. Linfante I, Akkawi NM, Perlow A, et al. Polyglycolide/polyactide-coated platinum coils for patients with ruptured and unruptured cerebral aneurysms: a single-center experience. Stroke 2005;36:1948-53. Epub 2005 Jul 28

10. Tashner CA, Leclerc X, Rachdi H, et al. Matrix detachable coils for the endovascular treatment of intracranial aneurysms: analysis of early angiographic and clinical outcomes. Stroke 2005;36:2176-80

11. Murayama Y, Vinuela F, Ishii A, et al. Initial clinical experience with Matrix detachable coils for the treatment of intracranial aneurysms. J Neurosurg 2006;105:192-99

12. Niimi Y, Song J, Madrid M, et al. Endosaccular treatment of intracranial aneurysms using Matrix coils: early experience and midterm follow-up. Stroke 2006;37:1028-32

13. Pierot L, Bonafé A, Bracard S, et al. Endovascular treatment of intracranial aneurysms with Matrix detachable coils: immediate posttreatent results from a prospective multicenter registry. AJNR Am J Neuroradiol 2006;27:1693-99

14. Tamatani S, Ito Y, Abe H, et al. Evaluation of the stability of aneurysms after embolization using detachable coils: correlation between stability of aneurysms and embolized volume of aneurysms. AJNR Am J Neuroradiol 2002;23:762-67

15. Ding YH, Dai D, Lewis DA, et al. Angiographic and histologic analysis of experimental aneurysms embolized with platinum coils, Matrix, and HydroCoil. AJNR Am J Neuroradiol 2005;26:1757-63

16. Gallas S, Pasco A, Cottier JP, et al. A multicenter study of 705 ruptured intracranial aneurysms treated with Guglielmi detachable coils. AJNR Am J Neuroradiol 2005;26:1723-31 